\title{
Damping Analysis of Plates with Multiple Constrained Layer Damping Treatments
}

\author{
Qingqing $\mathrm{Wu}$ \\ School of Marine Science and Technology \\ Northwestern Polytechnical University \\ 710072 Xi'an, China
}

\author{
Minqing Wang \\ School of Marine Science and Technology \\ Northwestern Polytechnical University \\ 710072 Xi’an, China
}

\begin{abstract}
In this paper, the damping analysis of plates with multiple constrained layer damping (CLD) treatments is presented. Based on the complex stiffness method, the theoretical damping model for multilayered constrained configurations is developed. Theoretical and experimental studies are conducted on the vibration damping characteristics of plates with different CLD treatments. It is shown that CLD can significantly improve the damping capacity of the substrate plate and deduce the vibration at middle and high resonant frequencies. The loss factor increase with increasing number of CLD treatments, while the vibration attenuation upon the increasing CLD treatment is not as apparent as the damping enhancement. The present study reveals how the damping capacity varies with multiple CLD treatments and will be in favor of structural design and vibration reduction of complex structures.
\end{abstract}

Keywords- Multiple Constrained; Constrained Layer Damping; Loss Factor; Viscoelastic.

\section{INTRODUCTION}

A constrained layer damping pair usually consists of a viscoelastic damping material layer and a constraining layer. Due to the constrained condition, the viscoelastic core undergo transverse shear damped deformation which produce more energy dissipation than free layer damping treatment. CLD can greatly enhance the damping property of structures and has been widely applied in aeronautics, astronautics and navigation industries.

As for the early work on CLD, Kerwin and Ungar [1,2] firstly developed the damping model of constrained configurations based on the complex stiffness method and subsequently proposed the strain energy method. Johnson and Kienholz [3] used the finite element method (FEM) to study the damping of constrained viscoelastic structures. Based on the complex moduli approach, Min Hao and Mohan D. Rao proposed a semi-analytical method to study the stiffness and damping of symmetrical viscoelastic beams with multiple CLD [4] and established the model to determine the dynamic properties of asymmetrical sandwich viscoelastic beams [5]. Torvik and Runyon [6] developed the R-U theory and proposed an approximate approach to determine the loss factors and frequencies of sandwich plates with CLD. Nowadays, the FEM have been widely applied to study complex structures. Carrera E [7] reviewed the theories and finite elements for multilayered, anisotropic, composite plates and shells. Based on the FEM, Masti and Sainsbury [8] analyzed the damping of cylindrical shells with partial CLD treatments; Kristensen R F and Nielsen K L [9] studied the shear damped composite beams with CLD; Venkatachalam R and Raja K V [10] investigated the effect of constrained layers on vibrational characteristics of a composite sandwich system. Benefiting from the self- adaptive and self-monitoring capabilities, active constrained layer damping (ACLD) has attracted much attention $[11,12]$. The vibration control and damping design of passive and active CLD treatments were studied numerically and experimentally [13-15].

The present study focuses on the damping capacity of plates with multiple CLD treatments. This study is helpful to better understand the effect of multiple CLD treatments on the damping capacity and can be applied in further optimization. In the theoretical study section, the theoretical damping model for multilayered constrained structures is developed based on the complex method. Simulations are accomplished with regard to a thick plate, investigating the effect of increasing CLD treatments. In the experimental study section, one thick plate is tested with different CLD treatments successively. Comparisons and discussions of the experimental results are presented to reveal how the CLD treatments effect the damping loss factor and the vibration response of plates.

\section{THEORETICAL STUDY}

\section{A. Theoretical modelling}

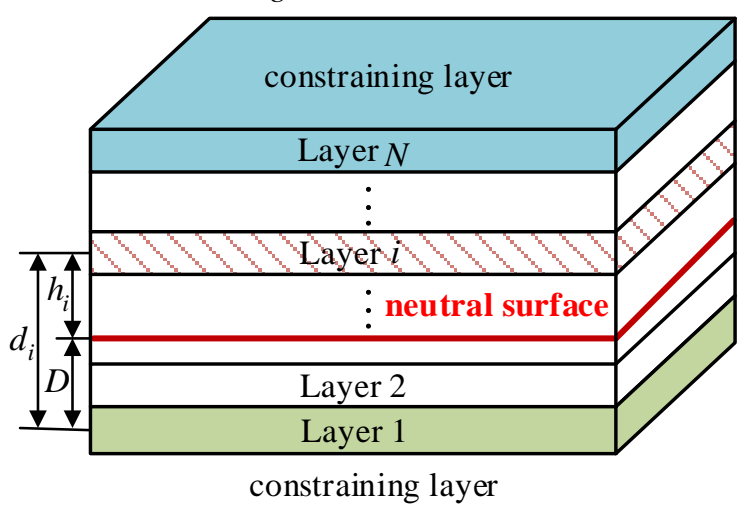

Fig. 1. Section sketch map of an $N$-layer CV laminate.

The sequence distribution of an N-layer constrained viscoelastic configuration is illustrated in Fig. 1, where $D$ is the distance from the neutral surface of the configuration to the neutral surface of the first layer; $d_{i}$ is the distance from the $i$ th layer's neutral surface to the center of the first layer; and $h_{i}$ is the distance from the neutral surface of an individual $i$ th layer to the neutral surface of the configuration. The face sheets are specified as constraining layers, while the interlayers can exhibit different material properties. 
The bending stiffness of the composite configuration can be expressed in the complex form

$$
\begin{gathered}
B^{*}=(E I)_{\text {re }}(1+\mathrm{i} \eta) \\
\alpha+\beta=\chi
\end{gathered}
$$

where $B^{*}$ is the complex stiffness, $(E I)_{\mathrm{re}}$ is the effective bending stiffness, and $\eta$ is the structure or system loss factor.

According to the relationship between moments and bending stiff nesses,

$$
B^{*} \frac{\partial \theta}{\partial x}=B_{\mathrm{o}} \frac{\partial \theta}{\partial x}+\sum_{i=1}^{N} F_{i} h_{i}
$$

where $B_{\mathrm{o}}=(E I)_{\mathrm{o}}=\sum_{i=1}^{N}(E I)_{i}$ is the sum of the individual layer's bending rigidities, $\theta$ is the rotate angle and $F_{i}$ is the net extensional force acting at the center of the $i$ th layer. Here $F_{i}=K_{i} \varepsilon_{i}$ where $\varepsilon_{i}$ and $K_{i}$ denote the strain in the center plane and the extensional stiffness, respectively.

Define $\phi=\partial \theta / \partial x, \varphi=\partial \gamma / \partial x$. Introduce the deformation parameter as

$$
R=\frac{\varphi}{\phi} H
$$

The deformation parameters can be determined by the equations as follows

$$
\begin{aligned}
& \frac{1}{K_{T}} \sum_{j=i}^{N} K_{j}\left[\frac{1}{K_{T}} \sum_{m=2}^{N}\left(\sum_{e=m}^{N} K_{e}-\frac{1}{2} K_{m}\right) R_{m}-\sum_{m=2}^{j} R_{m}+\frac{1}{2} R_{j}\right]-X_{i}^{*} R_{i} \\
& =\frac{1}{K_{T}} \sum_{j=i}^{N} K_{j}\left(\frac{1}{K_{T}} \sum_{m=2}^{N} K_{m} d_{m}-d_{j}\right)
\end{aligned}
$$

According to the mechanical analysis, the strain can be determined by

$$
\begin{gathered}
\varepsilon_{i}=\tilde{\varepsilon}_{i} \phi \\
\tilde{\varepsilon}_{i}=\left\{\begin{array}{l}
-\frac{1}{K_{\mathrm{T}}} \sum_{m=2}^{N} K_{m}\left(d_{m}+\sum_{e=1}^{m} R_{m}-\frac{1}{2} R_{i}\right) \quad(i=1) \\
d_{i}+\sum_{m=1}^{i} R_{m}-\frac{1}{2} R_{i}-\frac{1}{K_{\mathrm{T}}} \sum_{m=2}^{N} K_{m}\left(d_{m}+\sum_{e=1}^{m} R_{m}-\frac{1}{2} R_{i}\right) \\
(i=2,3, \ldots, N)
\end{array}\right.
\end{gathered}
$$

where $K_{\mathrm{T}}=\sum_{i=1}^{N} K_{i}$ is the sum of the individual extensional rigidities.

Define the coupling stiffness as $\tilde{B}=\sum_{i=2}^{N} K_{i} d_{i} \tilde{\varepsilon}_{i}$. Thus, (2) can be rewritten as

$$
B^{*}=B_{0}+\tilde{B}
$$

A dimensionless stiffness parameter is introduced as $W^{*}=\tilde{B} / B_{0}$. By combining (1) and (7), the effective bending stiffness and structure loss factor of the laminate can be obtained respectively as

$$
\begin{gathered}
(E I)_{\mathrm{re}}=B_{0}\left[1+\operatorname{Re}\left(W^{*}\right)\right] \\
\eta=\frac{\operatorname{Im}\left(W^{*}\right)}{1+\operatorname{Re}\left(W^{*}\right)}
\end{gathered}
$$

\section{B. Simulations and discussions}

In this section, three kinds of composite laminates with different CLD layers are considered. The composite structures have identical substrate steel plate with the thickness of $6 \mathrm{~mm}$. Three of them have identical stand-off layer (SOF) with the thickness of $6 \mathrm{~mm}$. The plates have one, two and three constrained layers, respectively. Each CLD is composed of 1mm-thick viscoelastic damping layer and 0.5 -mm-thick aluminum layer. The Young's modulus of the SOL is set to be $100 \mathrm{MPa}$ and the loss factor is set to be 0.2 . The loss factor and shear modulus of the viscoelastic material are as follows

$$
\log _{10} \beta=-1.2 \times\left(\log _{10} f\right)^{(-0.6)}+0.5
$$

$$
\log _{10} G=0.2 \times \log _{10} f+6
$$

The system loss factor of the composite plate is obtained by the present method. Loss factors of the plate with SOL in regard to three different CLD treatments are compared in Fig. 2. Loss factors of the plate without SOL in regard to three different CLD treatments are compared in Fig. 3.

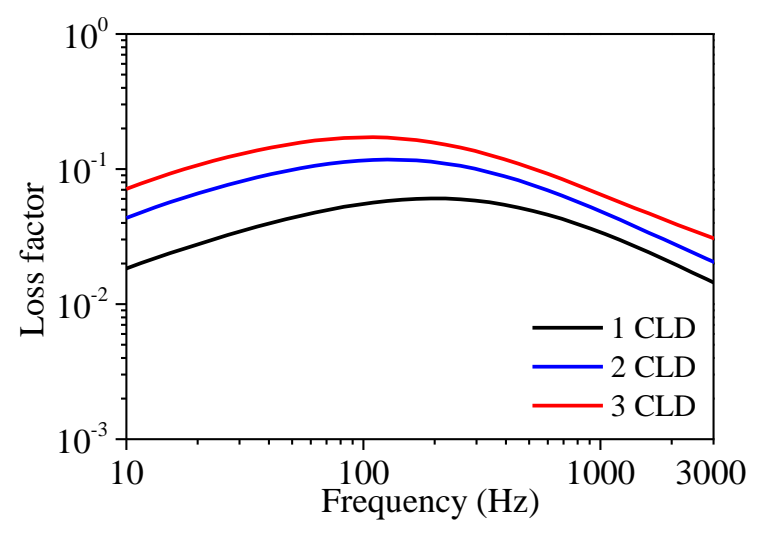

Fig. 2. Loss factor of the composite plate with SOL. 


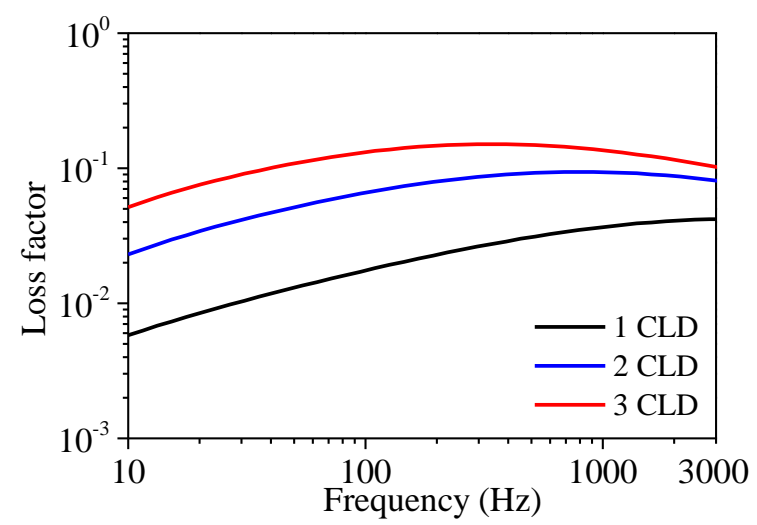

Fig. 3. Loss factor of the composite plate without SOL.

As shown in Fig. 2 and Fig. 3, the structure loss factor of the composite plate increases with increasing number of CLD treatments. For the thick plate in this study, the improvement of the damping capacity is more significant at low frequency. When comparing Fig. 2 with Fig. 3, it is shown that the introduction of stand-off layer improves the damping capacity at low frequency while deduce the loss factor at high frequency.

\section{EXPERIMENTAL STUDY}

\section{A. Experimental system}

To study the vibration damping characteristics of constrained plates, experiments are conducted on one thick plate with three different kinds of CLD treatments. The composite plate is $0.5 \mathrm{~m}$ wide and $0.6 \mathrm{~m}$ long with $6-\mathrm{mm}$-thick substrate steel plate and 7-mm-thick stand-off rubber layer. One to three pairs of CLD treatments are applied onto the stand-off layer, successively. The constraining layer is $0.1-\mathrm{mm}$ thick aluminum and the damping layer is 0.5 -mm-thick viscoelastic material.

The experimental system of the vibration damping measurement is illustrated in Fig. 4. As shown in Fig. 5, the tested plate is suspended with an elastic rope.

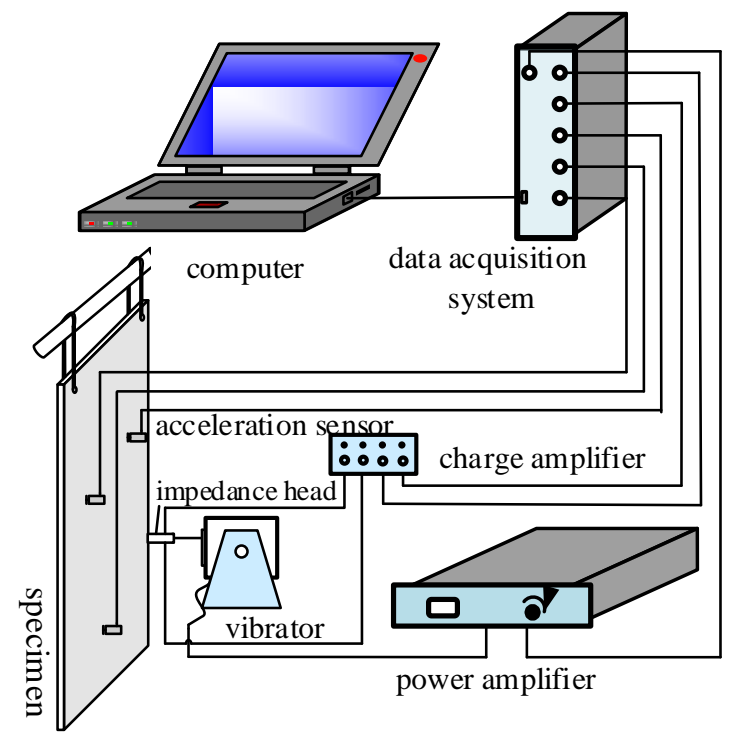

Fig. 4. Schematic diagram of the experimental apparatus.

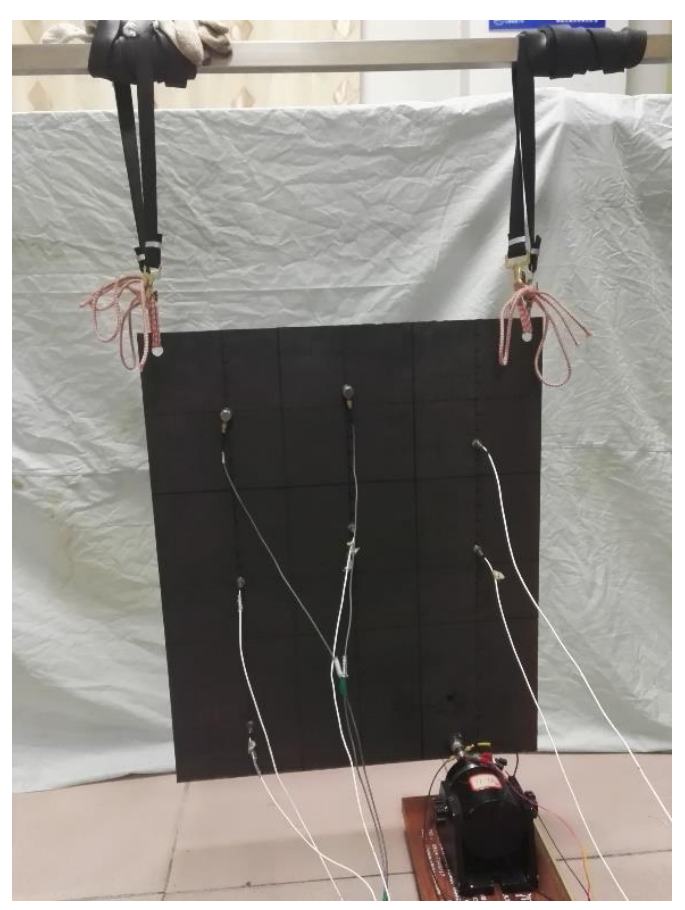

Fig. 5. Tested plate with constrained layer damping treatment.

\section{B. Test results and discussions}

The acceleration responses of several measurement points are obtained with vibrator excitation and the structure loss factor is obtained by the decay rate method with hammer impact. The loss factor of the substrate steel and structure loss factors of CLD treated plates are compared in Fig. 6. The acceleration responses for two measurement points are compared in Fig. 7.

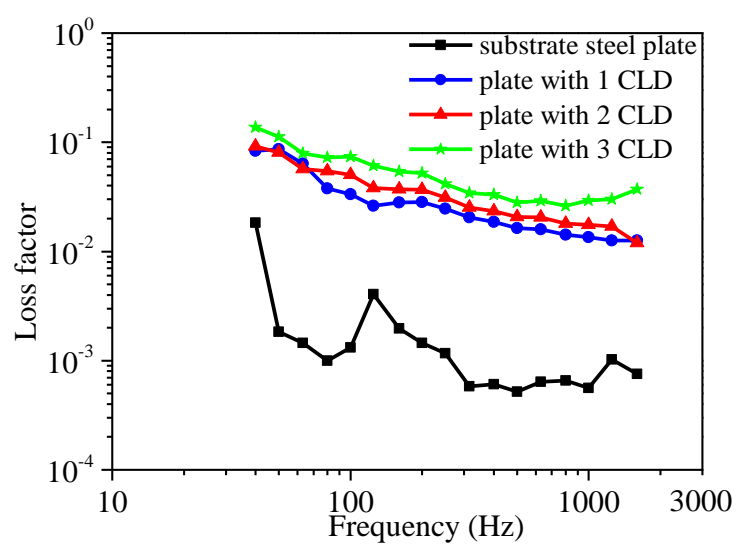

Fig. 6. Tested loss factor of the plate with different CLD treatments 


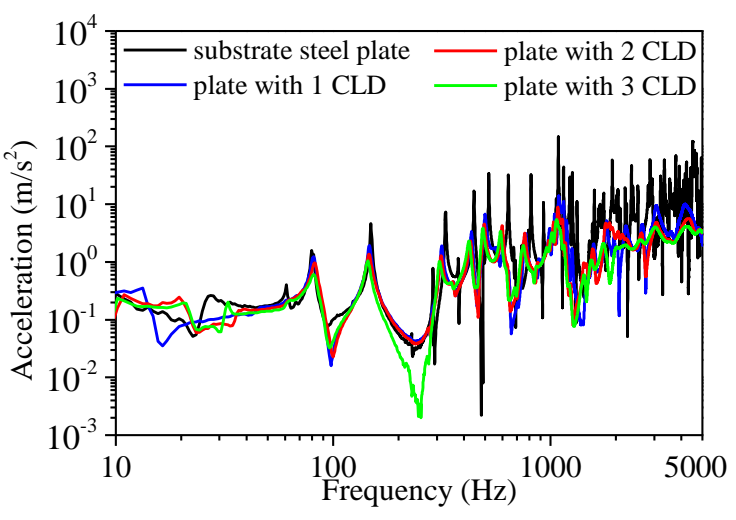

Fig. 7. Tested acceleration response of one test point with different CLD treatments

It can be seen from Fig. 6 that CLD can significantly improve the damping capacity of the plate, and the structural loss factor increases with increasing number of CLD treatments. Fig. 7 shows that CLD can greatly deduce the vibration at middle and high resonant frequencies while the effect is not obvious at low frequency. It can also be observed that the vibration attenuation upon the increasing number of CLD treatments is not as apparent as the increase of structure loss factor. It infers that a balance should be considered between the manufacturing cost and the damping capacity improvement.

\section{CONCLUSIONS}

Focus on the damping capacity of plates with multiple constrained layer damping (CLD) treatments, theoretical and experimental studies have been conducted. The damping enhance by the CLD treatments have been confirmed theoretically and experimentally. The structure loss factor increases with increasing number of CLD treatments. For the thick steel plate in this study, this improvement is more significant at low frequency. Test results have shown that CLD treatments can greatly deduce the vibration at middle and high resonant frequencies. However the vibration attenuation upon increasing CLD treatments is not as apparent as the damping gain. It infers that the balance of manufacturing cost and damping capacity optimization should be taken into account during the vibration attenuation design.
The present study investigates how damping and vibration varies with CLD treatments. It will be helpful for structural design and vibration reduction of complex structures.

\section{REFERENCES}

[1] Kerwin EM. Damping of flexural waves by a constrained viscoelastic layer. J Acoust Soc Am 1959; 31(7):952-962.

[2] Ungar EE, Kerwin EM. Loss factors of viscoelastic systems in terms of energy concepts. J Acoust Soc Am 1962; 34(7):954-957.

[3] Johnson CD, Kienholz DA. Finite element prediction of damping in structures with constrained viscoelastic layers. AIAA J 1982; 20:12841290 .

[4] Hao M, Rao MD, Schabus MH. Optimum Design of MultipleConstraint-Layered Systems for Vibration Control. AIAA J 2004 42(12):2448-2461.

[5] Hao M, Rao MD. Vibration and damping analysis of a sandwich beam containing a viscoelastic constraining layer. J Compos Mater 2005; 39(18):1621-1643.

[6] Torvik PJ, Runyon BD. Estimating the loss factors of plates with constrained-layer damping treatments. AIAA J 2007; 45(45):1492-1500

[7] Carrera E. Theories and finite elements for multilayered, anisotropic, composite plates and shells. J Arch Comput Methods Eng 2002; 9:87140

[8] Masti RS, Sainsbury M G. Vibration damping of cylindrical shells partially coated with a constrained viscoelastic treatment having a standoff layer. Thin Wall Struct 2005; 43(9):1355-1379.

[9] Kristensen RF, Nielsen KL, Mikkelsen LP. Numerical studies of shear damped composite beams using a constrained damping layer. Compos Struct 2008, 83(3):304-311.

[10] Venkatachalam R, Raja KV. Effect of constrained layers on vibrationa characteristics of a composite sandwich system-A finite element based critical investigation. INT J Mech Mater Des 2011; 7(4):291-298.

[11] Baz A. Optimization of energy dissipation characteristics of active constrained layer damping. Smart Mater Struct 1997;6:360.

[12] Vasques CMA, Mace BR, Gardonio P, et al. Arbitrary active constrained layer damping treatments on beams: Finite element modelling and experimental validation. Compu Struct 2006; 84(22):1384-1401.

[13] Inman DJ. Vibration control through passive constrained layer damping and active control. J Intel Mat Syst Str 1997; 8(8):663-677.

[14] Kumar N, Singh SP. Experimental study on vibration and damping of curved panel treated with constrained viscoelastic layer. Compos Struct 2010; 92:233-243.

[15] Kattimani S C, Ray M C. Vibration control of multiferroic fibrous composite plates using active constrained layer damping. Mech Syst Signal Pr 2018; 106:334-354. 